blood brain barrier developmental physiology erythrocytes

D-glucose

\author{
infants \\ plasma membrane \\ transfer \\ mechanisms
}

\title{
Kinetics of Glucose Transfer in Adult and Fetal Human Erythrocytes
}

\author{
Thomas Jenner Moore ${ }^{[19]}$ and Nancy Hall \\ Department of Pediatrics, St. Luke's Hospital Center, New York, New York, USA
}

\begin{abstract}
Extract
Efflux of D-glucose at $4^{\circ}$ was different in erythrocytes of healthy adults than it was in erythrocytes of term human infants. The calculated $K_{s}$ was $30.2 \mathrm{~mm}$ for the adult cells and $18.0 \mathrm{~mm}$ for the infant cells. The likeliest explanation of these results is the ontogeny of the sugar-carrier apparatus in the human erythrocyte.
\end{abstract}

\section{Speculation}

Evidence is presented that in the early stages of mammalian development transfer rates for certain solutes across the plasma membrane change substantially and that three of four possible transfer mechanisms participate in this change. This change may affect infant nutritional requirements and should be considered in establishing normal values of certain nutrients (e.g., glucose) in the premature and newly born. In addition, response of infants to drugs may be related to drug access to the target site. Whether other functions of the plasma membrane undergo developmental change in early postnatal life remains to be determined.

\section{Introduction}

Previous studies have demonstrated that the human fetal erythrocyte is different from the postnatal red cell. Differences with respect to size, survival time [II], and ability to swell [5] have been known for many years. More recently qualitative and quantitative differences in certain metabolic enzymes have been described. Some enzymes, e.g., serum glutamic oxalacetic transaminase (SGOT), are more active in young fetal cells than in young adult cells but lose this activity rapidly as the fetal cell matures. Other enzymes, such as hexokinase, exist as isoenzymes in fetal erythrocytes [12].

The membrane of the fetal erythrocyte also differs from its adult counterpart [4] in that it contains more sphingomyelin, less lecithin, and far less linoleic acid. Its transport properties also differ. Net potassium efflux is greater in the fetal cell [2], and glycerol penetrates less rapidly than in the adult cell [9].
This report describes efflux of D-glucose from adult and newborn erythrocytes and presents interpretive results in the context of knowledge about transport kinetics in early human development.

\section{Materials and Methods}

Venous blood from healthy adult volunteers was collected in heparinized tubes [17]. Samples of infant blood were aspirated from the umbilical stump of the placenta in the delivery room. Only infants estimated to be at full term by birth weight were used for these studies. All samples were studied immediately.

Infant and adult blood was centrifuged. The plasma and buffy coat were removed and the sedimented erythrocytes were resuspended in a roughly equal volume of $0.85 \% \mathrm{NaCl}$ adjusted with phosphate buffer to $\mathrm{pH} 7.4$ and recentrifuged. The saline wash was repeated three times. Reticulocyte counts on adult samples 
before centrifugation and after centrifugation and repeated washing averaged $1.35 \pm 0.37$ and $0.5 \pm 0.34 \%$, respectively. Reticulocyte counts on fetal samples before centrifugation and after centrifugation and washing averaged $4.12 \pm 1.3$ and $2.03 \pm 1.05 \%$, respectively.

\section{Glucose Efflux}

Experimental. The experimental procedure of Levine and Stein [8] was used. Briefly, the thrice-washed human erythrocytes were loaded with ${ }^{14} \mathrm{C}$-glucose $(20 \mu \mathrm{Ci} / \mathrm{g})$ [15] at varying total glucose concentrations. The packed cells were separated from the medium by centrifugation for $20 \mathrm{~min}$ at $4^{\circ}$. The loaded packed cells were then transferred to an ice bath and allowed to equilibrate separately with an unlabeled glucose solution. At zero time, $3.9 \mathrm{ml}$ unlabeled glucose were pipetted into $0.5 \mathrm{ml}$ packed loaded cells. The translocation was terminated by the addition of a ninefold volume of ice-cold stopping solution (2 $\mathrm{mM} \mathrm{HgCl}_{2}, 1.25 \mathrm{~mm} \mathrm{KI}$, in $0.7 \% \mathrm{NaCl}$ ) to the sample. An infinite time sample was obtained by equilibrating the loaded cells with medium for $40 \mathrm{~min}$ at room temperature before adding the stopping solution. The cells were immediately centrifuged, and the supernatant was discarded. The radioactive glucose remaining in the cells was extracted with trichloroacetic acid. Aliquots of the filtrate were transferred to scintillation vials containing $15 \mathrm{ml}$ Bray's solution and were counted in a spectrometer [16]. At least five determinations were done at timed intervals on each sample for the tracer efflux of D-glucose. Eleven samples at $0.0175 \mathrm{M}$, 10 at $0.025 \mathrm{M}, 8$ at $0.05 \mathrm{M}$, and 7 at $0.075 \mathrm{~m}$ glucose concentration were obtained for the points presented in Figure 2.

Theoretical. The equation describing tracer efflux into equilibrium sugar media of finite volume has already been derived [8]. It states

$$
\left[S_{i}^{R}(t)\right]=\frac{\alpha}{\beta}+\left(\left[S_{i}^{R}(O)\right]-\frac{\alpha}{\beta}\right) e^{-\beta t}
$$

where:

$$
\begin{aligned}
& S_{i} R(t)= \text { concentration of labeled sugar inside the cells } \\
& \text { at time } t \\
& S_{i}^{R}(O)= \text { concentration of labeled sugar outside the cells } \\
& \text { at time zero } \\
& \alpha= \frac{k_{2}[\text { tot } C] M^{R}}{2\left(K_{s}+[S]\right) V_{o}} \\
& \beta= \frac{V_{\max }, \text { equil. }\left(1+V_{i} / V_{o}\right)}{K_{s}+[\mathrm{S}]} \\
& k_{2}= \text { rate constant for the transfer across the mem- } \\
& \text { brane of the sugar-carrier complex }
\end{aligned}
$$

[tot $C]=$ concentration of total carrier

$M^{R}=$ total amount of labeled sugar on both sides of the membrane

$K_{\mathrm{s}}=$ sugar-site dissociation complex

$[S]=$ total sugar concentration

$V_{i}=$ volume of the compartment inside the cells

$V_{o}=$ volume of the compartment outside the cells

In the original derivation it was shown that $\left[S_{i}{ }^{R}(t)\right]$ diminishes to the limiting value $\left[S_{i}^{R}(\infty)\right]=\alpha / \beta$ as $t \rightarrow$ $\infty$. With data on concentration of radioactive sugar inside the cells at intervals of time and an infinite time sample, a plot of $\ln \left(\left[S_{i}^{R}(t)\right]-\alpha / \beta\right)$ against $t$ should give a straight line of slope $-\beta$. A second plot of $1 / \beta$ against $[S]$ will have an intercept equal to $K_{s} / V_{\max }$, equil. $\left(1+V_{i} / V_{0}\right)$ and a slope $1 / V_{\max }$, equil. ( $1+$ $\left.V_{i} / V_{o}\right)$. Hence $K_{s}$ can be calculated.

\section{Results}

Figure 1 is a plot of $\ln \left(\left[S_{i}^{R}(t)\right]-\alpha / \beta\right)$ against time in seconds at $0.0175 \mathrm{M}$ glucose concentration at $4^{\circ}$ for adult and infant cells. Both regressions are linear, but the slope for the infant data is steeper. Results for tracer efflux of $\mathrm{D}$-glucose in 11 experiments at $0.0175 \mathrm{M}$ are summarized in Table I. A significant difference is seen to exist between slopes of the disappearance of tracer for adult and for infant erythrocytes.

In Figure 2 the reciprocal of the slope is plotted against

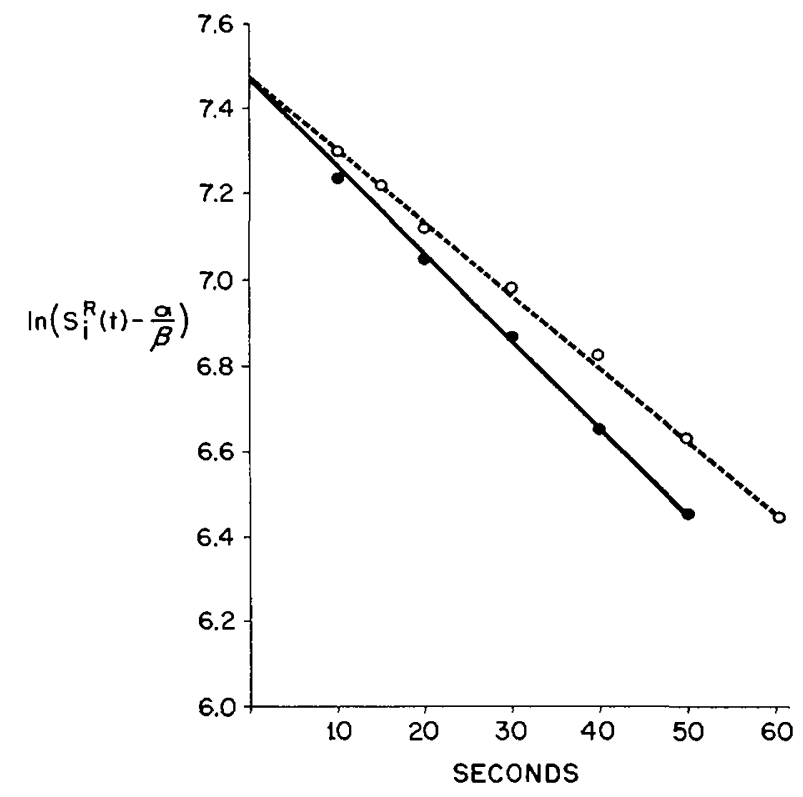

Fig. 1. Plot of $\ln \left(\left[S_{t}{ }^{R}(t)\right]-[\alpha / \beta]\right)$ against $t$ for the tracer efflux of ${ }^{14} \mathrm{C}$-D-glucose at $17.5 \mathrm{mM}$ total glucose concentration. $O$ : Course for adult blood. $\bullet$ : Course for infant blood. Temperature is $4^{\circ}$. The slope is Beta (see text). 
Table 1. Tracer efflux of D-glucose erythrocytes of adults and term infants ${ }^{1}$

\begin{tabular}{ccc}
\hline Erythrocytes & Reciprocal of regression & $P$ \\
\hline Adult & $58.6 \pm 1.9^{2}$ & 0.01 \\
Infant & $53.3 \pm 2.2$ & \\
\hline
\end{tabular}

${ }^{1}$ Glucose concentration was $17.5 \mathrm{~mm}$; temperature, $4^{\circ}$.

${ }^{2}$ Values are expressed as reciprocals to facilitate calculation of $K_{\text {s. }}$.

glucose concentration. Each point represents the mean of two to eight experiments. According to theory, the intercept on the ordinate is $K_{s} / V_{\max }$, equil. $\left(1+V_{i} / V_{o}\right)$ and the slope is $1 / V_{\max }$, equil. $\left(1+V_{i} / V_{o}\right)$. The $V_{\max }$, equil. appears from the data to be somewhat less in the infant cells. The intercept on the abscissa, which has the value of $-K_{\varepsilon}$, is seen to be substantially smaller in the infant cells. The calculated value for the adult cells is $30.2 \mathrm{~mm}$ and for the infant cells is $18.0 \mathrm{~mm}$. The adult value is in fairly good agreement with the value of 44 calculated elsewhere by this method at this temperature

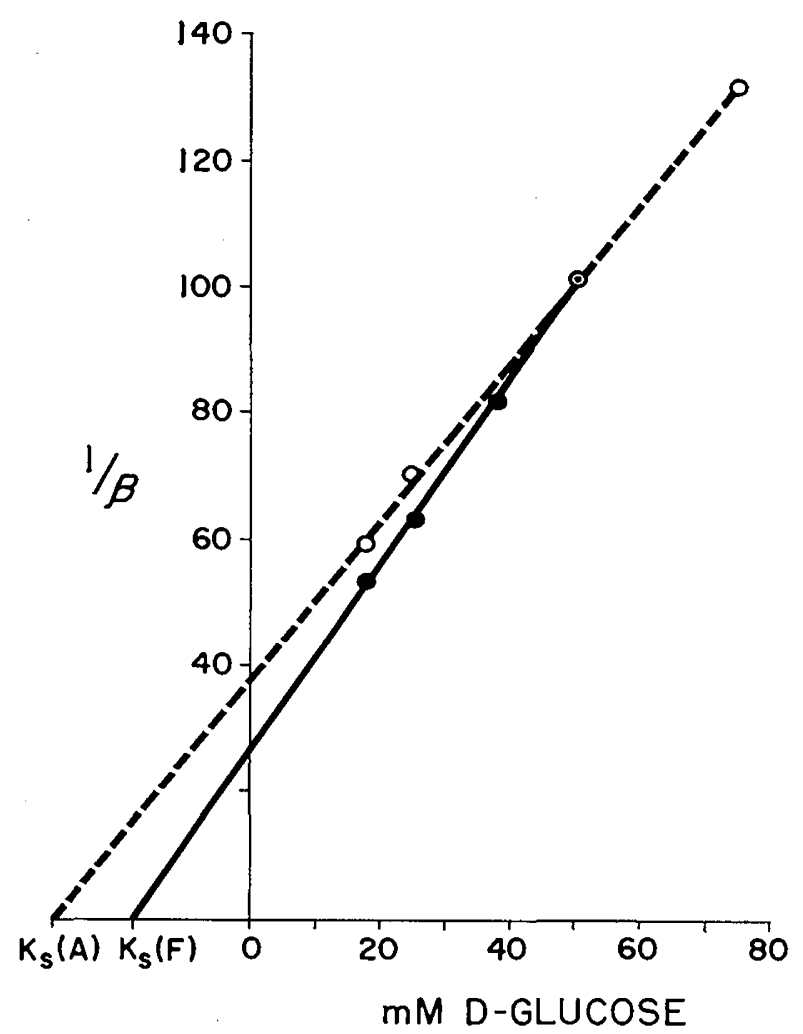

Fig. 2. Plot of 1/Beta (the reciprocal of the slope of tracer efflux) against $C$ at varying concentrations of total D-glucose. Temperature is $4^{\circ}$. 0 : Values for adult erythrocytes. $\bullet:$ Values for term infant erythrocytes. $F$ value of analysis of covariance for the two regressions is 21.39, $P<0.01$. The calculated $K_{s}$ values are $30.2 \mathrm{~mm} /$ liter adult and $18.0 \mathrm{~mm} /$ liter term fetal.
[8]. Three sources of error exist in these relations, and $K_{s}$ is calculated by extrapolation so that the values reported can only be considered as approximations. Nonetheless, the fact stands that substantial differences do exist in the sugar-site dissociation complex between the two populations. The infant cells have a greater affinity for the sugar.

\section{Discussion}

Sugar transport in the erythrocyte may be the simplest form of biological transport. Extensive investigations over the last 30 years have characterized many properties of this process [14]. Two properties are of consequence in this report. Sugar transport is not influenced by the presence of insulin. It is temperature-dependent and seems to be driven by thermal agitation, not by metabolic energy supplied by the cell. Therefore, it appears reasonable to attribute the measured differences in transport kinetics to fundamental differences in the erythrocyte membranes of adult and infant cells.

The fetal cell is macrocytic and therefore possesses a greater surface area and greater volume than the adult cell. The ratio of volumes to areas is roughly equal to the ratio of the radii raised to the $3 / 2$ power. Since the change in concentration of sugar inside the membrane during efflux varies inversely with the ratio of volume to area, these geometric differences predict a slower rate of sugar efflux in the fetal cell, all else being equal. Exactly the opposite was observed. Furthermore, it is held that sugar transport in the erythrocyte depends upon the existence of a limited number of transport sites on the cell membrane [14].

The samples studied were of different cellular ages. The infant samples were enriched with reticulocytes. Even after washing three times with saline prior to incubation in glucose, the infant reticulocyte count was $2.0 \pm 1.1 \%$, compared with a value of $0.5 \pm 0.3 \%$ for similarly treated adult cells. If the tracer efflux of glucose is, in fact, greater in a reticulocyte and this is what is responsible for the observed differences, then the tracer efflux decay curve should be monoexponential for the adult samples and a more complex function should be seen for the infant samples. Figure 1 shows that a monoexponential function best describes the tracer efflux for both samples.

Figure 1 and Table $I$ indicate that approximately a $10 \%$ difference in the rate of tracer efflux of glucose exists between the two populations at $0.0175 \mathrm{~m}$ concentration. The method measured disappearance of radioactive material from a space until about $15 \%$ of the initial content of label has escaped. Regardless of how rapidly tracer efflux of sugar takes place in the reticulocyte, if 
this cell type represents only $2 \%$ of the erythrocyte sample, it cannot by itself be responsible for the magnitude of the differences in slopes observed.

An alternative explanation is equally plausible. Bowyer [3] measured glucose penetration rates in erythrocytes of several mammalian species. In the sheep and guinea pig he found glucose readily penetrated the fetal cell but not the adult cell.

More recently, direct evidence for the ontogeny of membrane transport systems has been provided [1]. Renal iminoglycinuria, which occurs in the very young of both man and rat, was shown, in the rat, to be related to the absence of one of two distinct substrate-specific renal transport systems during the first 2 weeks of life.

Kupferberg and Way [6] have demonstrated that in the infant rat the change with age of morphine toxicity depends on altered drug uptake in the brain.

These three examples illustrate that the kinetics of penetration for certain solutes into a variety of tissues may be quite different in the very young mammal. Illustrated are three distinct mechanisms of penetration: mediated transport, active transport, and lipoid solubility.

The difference in mediated transport kinetics for $\mathrm{D}$ glucose reported here is held to be another illustration of the ontogeny of transport systems in the very young. It is unknown which transport system and which tissue is operative.

Renewed interest in sugar transport has brought to light certain interesting features. Regen et al. [13] have shown that glucose transport across the bloodbrain barrier in nephrectomized rats could be interpreted in terms of a mobile sugar carrier at the barrier, and that the $K_{m}$ for this process is $7 \mathrm{~mm}$, in close agreement with the $K_{m}$ for D-glucose entering adult human erythrocytes at $37^{\circ}$. In studying the penetration of glucose into the perfused rat liver, Regen et al. [13] found that the process was not affected by insulin and had the properties of mediated transport: stereospecificity, temperature dependence, and inhibition by phloridzin.

It seems likely that the molecular bases of sugar penetration into these three tissues share common properties. Attempts to isolate and characterize carrier apparatus have been limited to the erythrocyte. Earlier reports that fragments of ghosts resuspended in diethylaminoethyl cellulose column possessed sugar-carrier properties have not been confirmed [7]. Certain of the phospholipids found in the membrane of the erythrocyte have been shown to possess some of the physical properties ascribed to the hypothetical carrier apparatus, but by themselves they do not distinguish optical enantiomorphs [10]. The exact nature of the transport system thus remains uncertain.

\section{References and Notes}

1. Baerlocher, K., Monyuddin, F., AND SCRiver, C. R.: Ontogony of amino acid transport systems in mammalian kidney. Clin. Res., 18: 745 (1970).

2. BLUm, S. F., AND OsKI, F. A.: Red cell metabolism in the newborn infant. IV. Transmembrane potassium flux. Pediatrics, 43: 396 (1969).

3. BowYER, F.: The kinetics of penetration of nonelectrolytes into the mammalian erythrocyte. Int. Rev. Cytol., 6: 469 (1957).

4. Crowlex, J., Ways, P., AND Jones, J. W.: Human fetal erythrocyte and plasma lipids. J. Clin. Invest., 44: 989 (1965).

5. GUEST, G. M., AND WING, M.: Osmometric behavior of normal human erythrocytes. J. Clin. Invest., 21: 257 (1942).

6. Kupferberg, H. J., AND WAY, E. L.: Pharmacologic basis for the increased sensitivity of the newborn rat to morphine. J. Pharmacol. Exp. Ther., 141: 105 (1963).

7. LefeVre, P. G., AND MASIAK, S. J.: Lack of appreciable binding of D-glucose to red cell ghosts or ghost proteins. Amer. Chem. Soc. Nat. Meeting, Div. of Carbohydrate Chem., New York, Sept. 1969.

8. Levine, M., and Stein, W. D.: The kinetic parameters of the monosaccharide transfer system of the human erythrocyte. Biochim. Biophys. Acta, 127: 179 (1966).

9. MOORE, T. J.: Glycerol permeability of human fetal and adult erythrocytes and of a model membrane. J. Lipid Res., 9: 642 (1968).

10. MOORE, T. J.: Kinetic and equilibrium behavior of simple sugars in a water-butanol-lipid system. In: M. Blank: Surface Chemistry of Biological Systems (Pergamon Press, New York, 1970).

11. Oski, F. A., and Naiman, J. L.: Hematologic Problems in the Newborn, Chapt. 1 (Saunders, New York, 1966).

12. Oski, F. A., AND SMITH, D.: Red cell metabolism in the premature infant. III. Apparent inappropriate glucose consumption for cell age. Pediatrics, $41: 473$ (1968).

13. Regen, D. M., Buschiazzo, P. M., and Williams, T. F.: Glucose transport in liver and brain. Amer. Chem. Soc. Nat. Meeting, Div. of Carbohydrate Chem., New York, Sept. 1969.

14. Stein, W. D.: The Movement of Molecules across Cell Membranes, Chapt. 4 (Academic Press, New York, 1968).

15. Calbiochem, Los Angeles, Calif.

16. Model 4000, Packard Instrument Company, Downers Grove, III.

17. Informed consent was obtained prior to performing the venipuncture on the human subject in accordance with the provisions set forth in the Declaration of Helsinki.

18. Supported by Public Health Service Grant no. 04527-01 from the Division of Human Development, and by a New York Heart Association grant-in-aid.

19. Requests for reprints should be addressed to: Thomas J. Moore, M.D., Department of Pediatrics, St. Luke's Hospital Center, Amsterdam Avenue and 114th Street, New York, N. Y. 10025 (USA).

20. Accepted for publication October 21, 1970. 